\title{
Multiple Representations and Epistemic Games in Introductory Physics Exam Solutions
}

\author{
Adrienne L. Traxler ${ }^{*}$, , Jonathan V. Mahadeo ${ }^{\dagger}$, Daryl McPadden ${ }^{\dagger}$ and Eric Brewe** \\ * Department of Physics, Wright State University, 3640 Colonel Glenn Highway, Dayton, OH 45435 \\ ${ }^{\dagger}$ Department of Physics, Florida International University \\ ** Department of Teaching and Learning, Florida International University \\ 11200 SW 8th St, Miami, FL 33199
}

\begin{abstract}
Previous analysis of common exam questions in introductory physics at Florida International University has revealed differences in the number and type of epistemic games played by students in their solutions. Separated by course format (lecture/lab, lecture/lab/recitation, or inquiry-based), student work also shows varying use of multiple representational tools. Here we examine representation use in more detail to establish a descriptive picture of representation use across multiple instructors and course formats. We then compare these profiles with the epistemic games played by students, asking whether the same epistemic game shows the same pattern of representational tools across course types. We find that patterns of representation use vary by course format, but there are generally not clear representational "signatures" to uniquely identify epistemic games.
\end{abstract}

Keywords: epistemic games, representation use, problem solving, introductory physics

PACS: $01.40 . \mathrm{Fk}$

\section{INTRODUCTION}

Problem-solving is a broad skill studied in many STEM education contexts, and has been explored by physics education researchers as a central issue in the field [1]. Several frameworks have been fruitfully used in these studies, such as early expert-novice distinctions [2] and more recent expansions to include a transitional "journeyman" stage [3]. Two more recent and complementary lines of research are the study of student use of representations in physics $[4,5]$, and the description of students' construction of knowledge by playing epistemic "games" [6]. These latter two approaches have substantial overlap, in that representation use is a necessary part of playing epistemic games, and the game being played is likely to influence which representations students deploy. However, explicit comparisons of these two analysis frameworks are rare, and the first stage of such an analysis forms the subject of this paper.

Practicing scientists are fluent in a variety of representational tools, from introductory staples such as freebody diagrams to the specialized models and graphs that characterize particular subfields. Student use of such tools, and their flexibility with multiple formats on a given problem-solving task, have been charted in a variety of contexts. Meltzer [4] examines isomorphic quiz questions couched in different representational formats in introductory Physics 2, and also looks for gender differences. De Leone and Gire [7] consider the question of whether more representation use is invariably better, and find evidence that use of multiple representational tools is necessary but not sufficient on the quiz and exam problems they studied. Using a coding scheme adopted by the present study, Kohl and Finkelstein [5] examine representation use on exam problems and find that students in reform-based introductory physics sections show more flexible use of multiple representational tools.

Epistemic games and epistemic forms are a framework originally proposed by Collins and Ferguson [8] to describe the knowledge construction of scientists. Games include legal "moves" as well as a target epistemic form which guides the scientific inquiry or problem solution. Adapted by Tuminaro and Redish [6] to study the activity of physics students, the epistemic games framework allows us to sidestep the expert/novice dichotomy and provides a vocabulary to explore a more nuanced range of behavior among students.

How students select a particular path for problemsolving is not yet clear, and likely relates to their epistemological framing [3]. However, once they have (however implicitly) chosen an epistemic game to pursue, we can identify it by marking evidence of the "moves" made. Games may be very focused on sense-making, or may hew closely to algorithmic mathematical manipulations. For the coding used in the present work, we have taken the six introductory physics games originally proposed by Tuminaro and Redish [6], summarized in Table 1.

Some games have an embedded representational focus, as in the case of Pictorial Analysis, but many do not specify a particular representational signature. By attending to both the representation use and epistemic games strands of research, we aim to address the follow- 
TABLE 1. Summary of the six epistemic games used to classify student exam solutions in the data.

\begin{tabular}{|c|c|c|}
\hline Game & Name & Description \\
\hline 1 & $\begin{array}{l}\text { Mapping Meaning } \\
\text { to Mathematics }\end{array}$ & Students begin from conceptual understanding of the physical situation \\
\hline 2 & $\begin{array}{l}\text { Mapping Mathematics } \\
\text { to Meaning }\end{array}$ & Students develop a conceptual story from a physics equation \\
\hline 3 & Physical Mechanism & Students attempt to construct a coherent and descriptive story \\
\hline 4 & Pictorial Analysis & Students generate an external spatial representation \\
\hline 5 & Recursive Plug-and-Chug & $\begin{array}{l}\text { Students plug quantities into equations to produce numeric } \\
\text { answers, without conceptual understanding }\end{array}$ \\
\hline 6 & Transliteration to Mathematics & Students often use worked examples to develop solutions \\
\hline
\end{tabular}

ing questions:

1. What does overall student representation use look like on a common exam problem by course type?

2. Do the epistemic games played by students have a distinct representation use signature?

\section{METHODS}

Florida International University is a large, urban, public research university in south Florida. It is a Hispanicserving institution with a primarily commuter student body. Students in this analysis were enrolled in Physics 1 with calculus in the Fall 2011 semester. Six sections in all are included in the study: two of a traditional lecture/laboratory format (LL), one of a lecture/laboratory/recitation format (LLR) being piloted that semester, and three sections of an inquiry-based (IQB) format.

All six sections were given two common exam problems on their first semester final, and student solutions were scanned before being graded by their instructors. The problem presented here consisted of six subparts. We will focus on the third, where students were asked to calculate the mass of an object attached to an elastic cord, given stretched and unstretched cord length and a previously calculated spring constant. Summary information is given for student representation use on the problem as a whole, and representation use will be examined as a predictor of epistemic game played for the third subpart.

To record student representation use, we adopt the coding scheme of Kohl and Finkelstein [5]. Representation use is divided into graphical $(\mathrm{G})$, mathematical $(\mathrm{M})$, pictorial (P), and verbal (V) categories. In this study, only "extra" representations not necessary for a full-credit answer are noted for each subpart, as a binary (each type present/not present in a solution).

Student use of epistemic games was previously coded for the third subpart of the problem. We use the six games identified by Tuminaro and Redish [6] and summarized in Table 1 to describe student solutions. Mahadeo et al. [9] gives further detail about the coding process.
We use logistic regression to estimate the extent to which particular representational tools, or combinations of representations, predict the epistemic game played. Mathematical (M) representation use was required for the third subpart, so graphical (G), pictorial (P), and verbal $(\mathrm{V})$ types were thus available as extra representations. Logistic regression models were run with each game played as a binary outcome variable, predicted by the three extra representations as factors (present/not present) as well as interactions between those terms (so, for example, graphical+verbal might emerge as a significant indicator of a particular game). Although some previous work has reported gender differences in representation use [4], we choose not to attempt that here, as the sample sizes are already small for many representations and games.

\section{RESULTS}

Before looking for a specific representation/game link, we begin by describing overall patterns of student representation use in the data. Table 2 shows the frequency of extra representations not required to solve the problem, grouped by course type. Note that counts in this table are cumulative over the entire problem and thus some detail is lost (e.g., whether extra written representation or pictorial sketches were more common on a given subpart).

TABLE 2. Overall frequency of "extra" use of student representations, summed over all subparts and grouped by course type. Representations are graphical $(\mathrm{G})$, mathematical $(\mathrm{M})$, pictorial $(\mathrm{P})$, or verbal $(\mathrm{V})$.

\begin{tabular}{lrrrr}
\hline Course format & G & M & P & V \\
\hline LL $(\mathrm{N}=87)$ & 2 & 9 & 46 & 43 \\
LLR (N=135) & 5 & 7 & 47 & 50 \\
IQB (N=96) & 97 & 30 & 142 & 183 \\
\hline
\end{tabular}

Total enrollment varies between course types, so it is also illustrative to see the fraction of total possible representations that appeared. Figure 1 shows these values for the three course formats, normalizing for the num- 
ber of students in each format. We see that a larger proportion of students in the IQB sections used representations across the four representation categories, with LL and LLR generally lower and closer to each other in frequency.

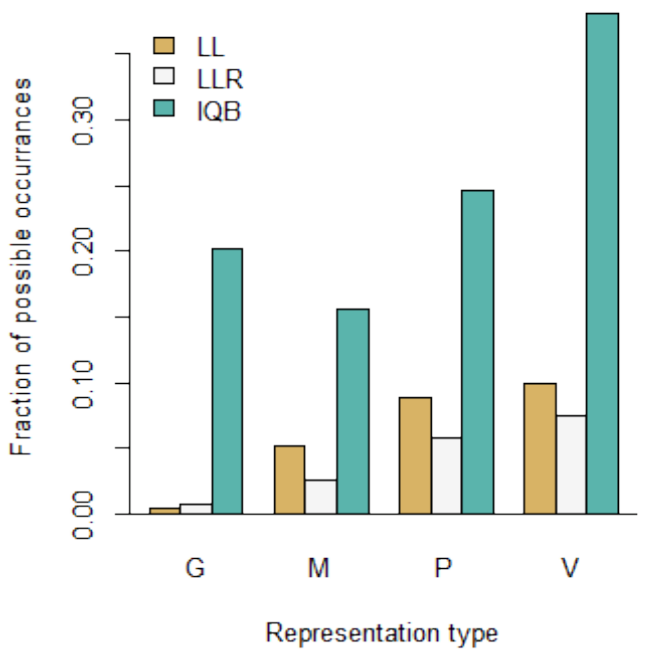

FIGURE 1. Overall relative frequency of "extra" representation use by course type, summed over the entire problem.

The third subpart of the problem will be examined in more detail to connect representation use with the epistemic games played by students. Figure 2 shows the relative frequency of different extra representation types on this portion of the problem.

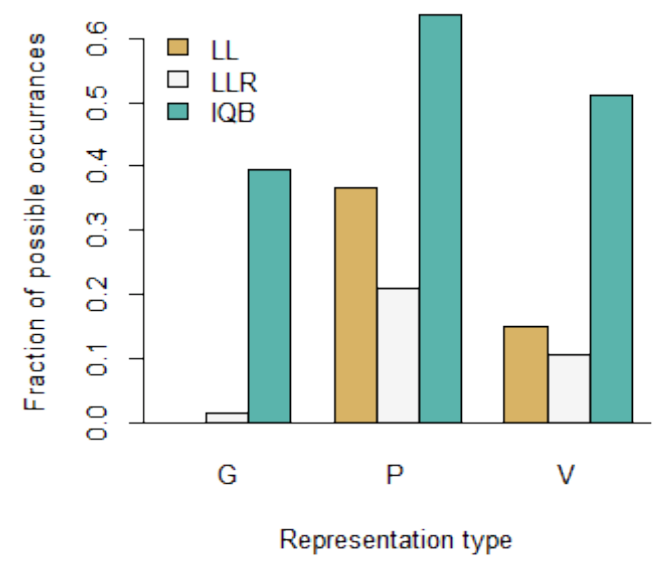

FIGURE 2. Frequency of "extra" representation use by course type on the third subpart of the problem. Mathematical (M) representation was required to complete this portion of the problem, thus does not appear as extra here.
Table 3 shows the results of the logistic regression models testing whether each epistemic game is tied to a particular set of representations. Marginal or no significance was found in the coefficients for games 1,5 , and 6 (Mapping Meaning to Mathematics, Recursive Plug and Chug, and Transliteration to Mathematics), indicating that no representations or combinations of them were signficant predictors of those games being played. Game 2 (Mapping Mathematics to Meaning) shows a significant coefficient for pictorial representation and a marginal interaction for pictorial with verbal. The signficant negative intercept indicates lower odds of playing this game for students who used no extra representations. Games 3 and 4 similarly show significant coefficients, although these should be taken cautiously, as relatively few students were found to play Physical Mechanism or Pictorial Analysis (perhaps because neither game, by itself, would yield the numerical answer required by the question).

\section{DISCUSSION}

To explore the link between the representations used and epistemic games played by students, it is first necessary to have a picture of each. Data on epistemic games already existed in our common exam question context from the analysis of Mahadeo et al. [9], but a corresponding picture of student representation use was lacking. Figures 1 and 2 provide this picture, for the exam problem as a whole and for the specific subpart compared with the epistemic games coding. At both levels of detail, we see that extra representations are more common in the reform-based class, which is consistent with earlier research findings [5] and with the generally greater emphasis placed on these tools in the reform curricula.

It is not entirely clear why the LL and LLR format classes seem to show some difference in the frequency of representation use. One possible explanation is that the LLR class, a new format in the Fall 2011 semester, may have had inconsistencies in the level of teaching assistant preparation or instructional emphasis in the recitation sections. These recitations were a redistribution rather than an addition of class time, so such inconsistencies might have led to less "time on task" in the LLR section for potentially helpful representation tools.

The larger question prompting this analysis is the extent to which representation use serves as a replacement or proxy for identifying the epistemic game played by a student. The former is a relatively quick analysis, conducted on this data for the entirety of both problems, while the epistemic game coding is more timeconsuming and was done here for only one subpart of one problem. Table 3 and accompanying discussion provide a first-order answer about the link: there is no clear "fin- 
TABLE 3. Logistic regression coefficients $\left(\beta_{i}\right)$ and standard errors (SE) modeling playing a game as outcome variable with representations as predictors. The constant term represents the coefficient for the odds of playing each game when only the default mathematical (M) representation is present. Only starred coefficients are significant at the $p<0.05$ level. In some cases, the combination of small $N$ for a game with sparsity of representation use produces large standard errors. Game 1 (which has no statistically significant coefficients) is omitted entirely from the table for this reason.

\begin{tabular}{lcccccrrrrr} 
& $\begin{array}{c}\text { Game 2 } \\
\text { Mapping Meaning } \\
\text { to Math }\end{array}$ & \multicolumn{2}{c}{$\begin{array}{c}\text { Game 3 } \\
\text { Physical } \\
\text { Mechanism }\end{array}$} & \multicolumn{2}{c}{$\begin{array}{c}\text { Game 4 } \\
\text { Pictorial } \\
\text { Analysis }\end{array}$} & $\begin{array}{c}\text { Game 5 } \\
\text { Recursive } \\
\text { Plug and Chug }\end{array}$ & $\begin{array}{c}\text { Game 6 } \\
\text { Transliteration } \\
\text { to Mathematics }\end{array}$ \\
\hline Term & \multicolumn{1}{c}{$\beta_{i}$} & $\mathrm{SE}$ & \multicolumn{1}{c}{$\beta_{i}$} & $\mathrm{SE}$ & \multicolumn{1}{c}{$\beta_{i}$} & $\mathrm{SE}$ & $\beta_{i}$ & $\mathrm{SE}$ & $\beta_{i}$ & $\mathrm{SE}$ \\
\hline (Const.) & $-2.2^{* * *}$ & 0.28 & $-4.9^{* * *}$ & 1.0 & $-4.2^{* * *}$ & 0.71 & $-0.30 \dagger$ & 0.18 & -0.15 & 0.17 \\
$\mathrm{G}$ & -13 & 840 & $4.2^{* *}$ & 1.58 & -12 & 1400 & -0.39 & 1.2 & -0.54 & 1.2 \\
$\mathrm{P}$ & $0.90^{*}$ & 0.41 & -16 & 2200 & $1.9 *$ & 0.83 & 0.078 & 0.31 & -0.34 & 0.31 \\
$\mathrm{~V}$ & 0.85 & 0.54 & $3.36^{* *}$ & 1.1 & 0.89 & 1.24 & -0.62 & 0.45 & -0.14 & 0.42 \\
$\mathrm{G} \times \mathrm{P}$ & 16 & 840 & -4.2 & 5200 & 14 & 1400 & -0.68 & 1.4 & -1.5 & 1.6 \\
$\mathrm{G} \times \mathrm{V}$ & 16 & 840 & $-3.4 \dagger$ & 1.9 & 14 & 1400 & -0.30 & 1.7 & -16.7 & 1600 \\
$\mathrm{P} \times \mathrm{V}$ & $-1.5 \dagger$ & 0.88 & 15 & 2200 & -1.7 & 1.7 & 0.21 & 0.68 & 0.54 & 0.65 \\
$\mathrm{G} \times \mathrm{P} \times \mathrm{V}$ & -15 & 840 & 2.8 & 5200 & -13 & 1400 & -0.48 & 2.2 & 1.3 & 2000 \\
\hline
\end{tabular}

${ }^{* * *} p<0.001 .{ }^{* *} p<0.01 .{ }^{*} p<0.05 . \dagger p<0.1$.

gerprint" connecting a particular epistemic game with a set of representations. Some suggestive elements appear, such as the appearance of a significant coefficient for pictorial representation (a sketch of the motion being analyzed, motion map, free-body diagram, etc.) for the popular Mapping Mathematics to Meaning game. In general, however, we find scant statistical link between the supplemental tools used by a student and the epistemic game they played. The two analyses, while they share some overlap, yield different insights about student problemsolving and cannot be regarded as substitutes for each other.

Several possible directions for future work may proceed from this study. One is to extend the analysis to more data, generating representation use and epistemic game codes for more problem solutions. This extension could be done with similar student populations or the same, examining other problem subparts or the second common exam question from the Fall 2011 semester. It is also possible that the relaxed time constraints offered by homework problems might reveal a greater variety of representation use as students took time to try alternate approaches. This line of work would address the small-N problem of games 1,3 , and 4, and explore whether our results generalize to a wider range of problem tasks.

A second direction for analysis is to take a more finegrained look at the representations that were used, the games played, and student performance on the problem. Much of the work on epistemic games in physics is descriptive and exploratory, identifying these knowledgeconstructing processes but not linking them to other outcomes such as quality of a solution or flexible navigation between epistemological frames [3]. Much as De Leone and Gire [7] found student use of representational tools "necessary but not sufficient" to a successful problem solution, we may find that accurate (rather than merely present) representations are a stronger signature for particular games, or that facility with particular games leads to more desirable student learning outcomes.

\section{ACKNOWLEDGEMENTS}

The authors thank the PER Group at FIU for their feedback. This research is supported by HHMI grant \# 52006924 \& NSF grant \# 0802184.

\section{REFERENCES}

1. L. Hsu, E. Brewe, T. M. Foster, and K. A. Harper, American Journal of Physics 72, 1147 (2004).

2. J. Larkin, J. McDermott, D. P. Simon, and H. A. Simon, Science 208, 1335-1342 (1980), ISSN 0036-8075.

3. T. J. Bing, and E. F. Redish, Physical Review Special Topics - Physics Education Research 8, 010105 (2012).

4. D. E. Meltzer, American Journal of Physics 73, 463-478 (2005).

5. P. B. Kohl, and N. D. Finkelstein, Physical Review Special Topics - Physics Education Research 2, 010102 (2006).

6. J. Tuminaro, and E. F. Redish, Physical Review Special Topics - Physics Education Research 3, 020101 (2007).

7. C. J. De Leone, and E. Gire, "Is Instructional Emphasis on the Use of Non-Mathematical Representations Worth the Effort?," in 2005 Physics Education Research Conference, edited by P. Heron, L. McCullough, and J. Marx, 2006, vol 818 of AIP Conference Proceedings, pp. 45-48.

8. A. Collins, and W. Ferguson, Educational Psychologist 28, 25-42 (1993), ISSN 0046-1520.

9. J. V. Mahadeo, A. L. Traxler, and E. Brewe, "Epistemic games analysis of common exam questions across course formats," in 2013 Physics Education Research Conference, edited by P. V. Engelhardt, A. D. Churukian, and D. L. Jones, Portland, OR, 2014. 